\title{
TOEPLITZ OPERATORS ON THE SEGAL-BARGMANN SPACE
}

\author{
C. A. BERGER AND L. A. COBURN
}

\begin{abstract}
In this paper, we give a complete characterization of those functions on $2 n$-dimensional Euclidean space for which the Berezin-Toeplitz quantizations admit a symbol calculus modulo the compact operators. The functions in question are characterized by a condition of "small oscillation at infinity".
\end{abstract}

1. Introduction. We consider the Toeplitz operators on the Segal-Bargmann space $H^{2}\left(\mathbf{C}^{n}, d \mu\right)$ of Gaussian square-integrable entire functions on $\mathbf{C}^{n}$. Such operators have been studied by Berezin and others $[\mathbf{4}, \mathbf{5}, \mathbf{1 0}, \mathbf{1 1}]$ and arise naturally as "anti-Wick quantization operators". Via the Schrödinger representation $[\mathbf{7}, \mathbf{1 0}]$, there is a natural equivalence between Topelitz operators on $H^{2}\left(\mathbf{C}^{n}, d \mu\right)$ and a generalization of pseudodifferential operators on $L^{2}\left(\mathbf{R}^{n}, d v\right)$, the so-called Weyl quantization $[\mathbf{9}, \mathbf{1 0}]$.

Let $P$ be the orthogonal projection operator $L^{2}\left(\mathbf{C}^{n}, d \mu\right)$ onto $H^{2}\left(\mathbf{C}^{n}, d \mu\right)$ with $d \mu(z)=(2 \pi)^{-n} e^{-|z|^{2} / 2} d v(z)$ and $d v(z)$ ordinary Lebesgue measure on $\mathbf{C}^{n}$. For $f$ in $L^{\infty}\left(\mathbf{C}^{n}\right)$, the multiplication operator $M_{f}$ on $L^{2}\left(\mathbf{C}^{n}, d \mu\right)$ is defined by $M_{f} h=f h$. The Toeplitz operator $T_{f}$ is defined, for $h$ in $H^{2}\left(\mathbf{C}^{n}, d \mu\right)$, by

$$
T_{f} h=P M_{f} h=P(f h) .
$$

In this paper, we complete the program, begun in [6], of determining the largest *-algebra $Q$ in $L^{\infty}\left(\mathbf{C}^{n}\right)$ for which $T_{f} T_{g}-T_{f g}$ is a compact operator for all $f, g$ in $Q$. Functions in $Q$ are characterized by a condition of "small oscillation at infinity".

It should be noted that the Weyl unitary operators [7] which generate the SegalBargmann representation of the Heisenberg group on $\mathbf{C}^{n}[\mathbf{1}, \mathbf{1 4}, \mathbf{1 5}]$ take the form $W_{\lambda}=T_{e_{\lambda}(z) \exp \left\{|\lambda|^{2} / 4\right\}}$ for $\lambda$ in $\mathbf{C}^{n}$, where [6] $e_{\lambda}(z)=\exp \{i \operatorname{Im} \bar{\lambda} \cdot z\}$ and $\bar{\lambda} \cdot z=$ $\bar{\lambda}_{1} z_{1}+\bar{\lambda}_{2} z_{2}+\cdots+\bar{\lambda}_{n} z_{n}$ for $z=\left(z_{1}, z_{2}, \ldots, z_{n}\right)$ in $\mathbf{C}^{n}$ and $|\lambda|^{2}=\left|\lambda_{1}\right|^{2}+\left|\lambda_{2}\right|^{2}+$ $\cdots+\left|\lambda_{n}\right|^{2}$. Thus, the $C^{*}$-algebra $C C R\left(\mathbf{C}^{n}\right)$ generated by the $\left\{W_{\lambda}\right\}$ is just the closure, in the operator norm, of

$$
\left\{T_{f}: f \text { a trigonometric polynomial on } \mathbf{C}^{n}=\mathbf{R}^{2 n}\right\}
$$

[6]. Since $C C R\left(\mathbf{C}^{n}\right)$ is known to be simple [7], nonconstant trigonometric polynomials cannot be in $Q$. On the other hand, we shall see that $Q$ contains all functions, such as $e^{i \sqrt{|z|}}$, which oscillate "less than linearly". We shall also show that $Q$ is closely related to $C C R\left(\mathbf{C}^{n}\right)$ in a more direct way.

Received by the editors May 23, 1986.

1980 Mathematics Subject Classification (1985 Revision). Primary 47B35; Secondary 81D05.

Research supported by grants of the National Science Foundation. 
For a precise statement of the main results, we require several definitions

$$
\begin{aligned}
& \Gamma=\left\{f \in L^{\infty}\left(\mathbf{C}^{n}\right): H_{f} \equiv(I-P) M_{f} P \text { is compact }\right\} \\
& B=\left\{f \in L^{\infty}\left(\mathbf{C}^{n}\right): P M_{f} P \text { is compact }\right\}
\end{aligned}
$$

We also have the function algebras:

$$
\begin{aligned}
E S V & =\left\{f \in L^{\infty}\left(\mathbf{C}^{n}\right): \operatorname{Lim}_{\substack{R \rightarrow \infty \\
|z-w| \leq 1 \\
|z| \geq R}}|f(z)-f(w)|=0\right\}, \\
V & =\left\{f \in L^{\infty}\left(\mathbf{C}^{n}\right): \operatorname{Lim}_{|z| \rightarrow \infty} f(z)=0\right\} \\
B C & =\text { Bounded continuous functions on } \mathbf{C}^{n} .
\end{aligned}
$$

We ignore sets of measure zero in the above definitions of $E S V$ and $V$. We write $B C E S V=B C \cap E S V$ and $C_{0}=B C \cap V$.

For $f$ in $L^{\infty}\left(\mathbf{C}^{n}\right)$, we make use of the convolution transform

$$
\tilde{f}(a)=(2 \pi)^{-n} \int f(z) e^{-|z-a|^{2} / 2} d v(z) .
$$

This transform is the Berezin symbol of the operator $T_{f}[\mathbf{3}]$ and is also the solution of the heat equation on $\mathbf{C}^{n}=\mathbf{R}^{2 n}$ at time $t=\frac{1}{2}$ with initial values $f[\mathbf{4}, \mathbf{8}, \mathbf{1 0}]$.

Let $K$ denote the ideal of all compact operators on the relevant Hilbert space, $H$. Let $\pi$ be the usual quotient map from $B(H)$ onto $B(H) / \mathcal{K}$ where $B(H)$ is the algebra of all bounded operators on $H$. We denote by $\tau(Q)$ the $C^{*}$-algebra generated by all $T_{f}$ with $f$ in $Q$.

Our main results can now be summarized.

Proposition A. $Q=\left\{f \in L^{\infty}\left(\mathbf{C}^{n}\right):(I-P) M_{f} P\right.$ and $(I-P) M_{\bar{f}} P$ are compact $\}$. For $f$ in $Q, T_{g} T_{f}-T_{g f}$ and $T_{f} T_{g}-T_{g f}$ are in $\mathcal{K}$ for all $g$ in $L^{\infty}\left(\mathbf{C}^{n}\right)$. $Q$ is the unique maximal *-subalgebra of $L^{\infty}\left(\mathbf{C}^{n}\right)$ with the property that $T_{f} T_{g}-T_{f g}$ is compact for all $f, g$ in $Q$.

THEOREM B. $(I-P) M_{f} P$ is compact if and only if $(I-P) M_{\bar{f}} P$ is compact. Moreover, $Q=\Gamma=E S V+Q \cap B$.

THEOREM C. The ideal $Q \cap B$ is given by $Q \cap B=\left\{f \in L^{\infty}\left(C^{n}\right): \widetilde{|f|^{2}} \in C_{0}\right\}$.

THEOREM D. The commutant of $\pi\left\{C C R\left(\mathbf{C}^{n}\right)\right\}$ in $B\left[H^{2}\left(\mathbf{C}^{n}, d \mu\right)\right] / K$ is $\pi\{\tau(Q)\}$. Equivalently, $\left[A, W_{\lambda}\right]$ is in $\mathcal{K}$ for all $\lambda$ in $\mathbf{C}^{n}$ if and only if $A-T_{f}$ is in $\mathcal{K}$ for some $f$ in $E S V$.

THEOREM E. $\pi\{\tau(Q)\} \simeq Q / Q \cap B \simeq E S V / V \simeq B C E S V / C_{0}$.

It should be pointed out that the algebra $Q$ is the homolog of the algebra $Q C$ of quasi-continuous functions in the case of Toeplitz operators on the unit circle. Moreover, $\Gamma$ is the homolog of the algebra $H^{\infty}+C$. Of course, on the circle, $Q C \neq H^{\infty}+C$. The absence of nonconstant bounded entire functions on $\mathbf{C}^{n}$ seems to be reflected in the fact that $Q=\Gamma$.

A critical ingredient in our analysis is an averaging operation over the SegalBargmann representation of the Heisenberg group given, for $A$ in $B\left\{H^{2}(d \mu)\right\}$, by

$$
\hat{A}=\int W_{\lambda}^{*} A W_{\lambda} d \mu(\lambda) .
$$


In Theorem 6 of $\S 3$, we discuss some useful properties of $\hat{A}$ and relate $\hat{A}$ to the Berezin symbol $\tilde{A}[\mathbf{3}]$.

We recall that $H^{2}(d \mu)$ has the reproducing kernels $e^{\tilde{a} \cdot z / 2}$ so, for $g$ in $H^{2}(d \mu)$,

$$
g(a)=\left\langle g, e^{\tilde{a} \cdot z / 2}\right\rangle=(2 \pi)^{-n} \int g(z) e^{a \cdot \bar{z} / 2} e^{-|z|^{2} / 2} d v(z) .
$$

Normalizing, we have $k_{a}(z)=e^{\bar{a} \cdot z / 2-|a|^{2} / 4}$ with $\left\|k_{a}\right\|=1$ in $H^{2}(d \mu)$. In terms of the $k_{a}$, the Berezin symbol of any operator $A$ on $H^{2}(d \mu)$ is defined by [3]

$$
\tilde{A}(a)=\left\langle A k_{a}, k_{a}\right\rangle \text {. }
$$

It is known that $\tilde{A}(a)$ is a smooth function which is uniquely determined by $A$. Moreover, it is not hard to check that $\tilde{A}$ is in $C_{0}$ for $A$ compact and, for all bounded $A, \hat{A}=T_{\tilde{A}}$ where $\hat{A}$ is the average over the Heisenberg group defined above.

Let $\tilde{f}^{(m)}$ denote the $m$ th iterate of $\tilde{f}$. The main idea in the proofs of Theorems B, C, D, E is to note that $E S V$ is characterized by $f-\tilde{f}^{(m)} \in V$ for all $m>0$ and use the fact that $\tilde{f}^{(m)}$ is Lipschitz with modulus of continuity converging to 0 as $m \rightarrow \infty$. We also use the fact that $\int K(a) d \nu(a)$ is compact whenever $K(a)$ is a uniformly bounded weakly measurable compact operator valued function and $\nu$ is a positive measure of finite total mass.

We remark that $T_{f}$ is bounded for $f$ in a larger class than $L^{\infty}$. In particular, $M_{f} P$ is bounded if $f$ is measurable and $\widetilde{|f|^{2}}$ is bounded.

In $\S 2$ of this paper, we discuss some analytic preliminaries. The functiontheoretic properties of $\tilde{f}$ are discussed and the class $E S V$ is described in terms of $\tilde{f}$. In $\S 3$, Theorems B, C, D are proved. In $\S 4$, the algebra $\tau(Q)$ is analyzed using earlier results. The index theory of $\tau(Q)$ is described. Finally, in $\S 5$, we discuss extensions and generalizations.

We thank William Arveson, Rodger Howe, Richard Rochberg, Irving Segal and William Zame for useful advice and discussions.

2. Preliminary results. We now discuss some analytic preliminaries. Beyond the definitions in $\S 1$, we will use the space

$$
\Lambda(\varepsilon)=\{f \in B C:|f(a)-f(b)| \leq \varepsilon|a-b|, \text { all } a, b\} .
$$

We note that $\Gamma, B, Q, E S V, V, B C, C_{0}$ are all closed. $V$ is an ideal in $L^{\infty}$ and $C_{0}$ is an ideal in $B C$. ESV and $B C$ are conjugate-closed algebras. It is easy to check, as in $[6]$, that $\Gamma$ is an algebra and that $B$ is a $\Gamma$ module so that $\Gamma \cap B$ is an ideal in $\Gamma$.

We begin by sketching the proof of

Proposition A. $Q=\left\{f \in L^{\infty}:(I-P) M_{f} P\right.$ and $(I-P) M_{\bar{f}} P$ are compact $\}$. For $f$ in $Q, T_{g} T_{f}-T_{g f}$ and $T_{f} T_{g}-T_{g f}$ are in $\mathcal{K}$ for all $g$ in $L^{\infty} . Q$ is the unique maximal *-subalgebra of $L^{\infty}\left(\mathbf{C}^{n}\right)$ with the property that $T_{f} T_{g}-T_{f g}$ is in $\mathcal{K}$ for all $f, g$ in $Q$.

PROOF. See [6]. For completeness, note that

$$
\begin{aligned}
P M_{g f} P & =P M_{g}\{P+(I-P)\} M_{f} P \\
& =\left(P M_{g} P\right)\left(P M_{f} P\right)+P M_{g}\left\{(I-P) M_{f} P\right\} .
\end{aligned}
$$


It follows at once that $T_{g} T_{f}-T_{g f}$ is in $\mathcal{K}$ for $f$ in $\Gamma$ and $g$ in $L^{\infty}$. On the other hand

$$
\left\{(I-P) M_{g} P\right\}^{*}\left\{(I-P) M_{g} P\right\}=P M_{|g|^{2}} P-\left(P M_{\bar{g}} P\right)\left(P M_{g} P\right)
$$

so $T_{\bar{g}} T_{g}-T_{|g|^{2}}$ is in $\mathcal{K}$ if and only if $g$ is in $\Gamma$. The desired result follows at once.

For $d \mu(z)=(2 \pi)^{-n} e^{-|z|^{2} / 2} d v(z)$ on $\mathbf{C}^{n}$, we recall that the subspace $H^{2}(d \mu)$ consists of all entire functions in $L^{2}(d \mu)$. For $g$ in $H^{2}(d \mu)$, we have the reproducing kernels $e^{\bar{a} \cdot z / 2}$ with

$$
g(a)=\left\langle g(z), e^{\bar{a} \cdot z / 2}\right\rangle=(2 \pi)^{-n} \int g(z) e^{a \cdot \bar{z} / 2} e^{-|z|^{2} / 2} d v(z) .
$$

If $P$ is the orthogonal projection operator from $L^{2}(d \mu)$ onto $H^{2}(d \mu)$ it follows that, for $b$ in $L^{2}(d \mu)$

$$
(P b)(z)=(2 \pi)^{-n} \int b(w) e^{z \cdot \bar{w} / 2} e^{-|w|^{2} / 2} d v(w) .
$$

Denoting by $M_{f}$ the operator of "multiplication by $f$ " on $L^{2}(d \mu)$, we will need to estimate the norms of $\left[M_{f}, P\right]=M_{f} P-P M_{f}$ and $P M_{|f|^{2}} P$. Such estimates can be obtained by using the unitary map from $L^{2}(d \mu)$ to $L^{2}\left((2 \pi)^{-n} d v\right)$ given by

$$
(U g)(z)=e^{-|z|^{2} / 4} g(z)
$$

Proposition 1. For $b$ in $L^{2}\left((2 \pi)^{-n} d v\right)$,

$$
\begin{gathered}
U\left[M_{f}, P\right] U^{*} b(z)=(2 \pi)^{-n} \int k(f, z, w) b(w) d v(w), \\
U P M_{|f|^{2}} P U^{*} b(z)=(2 \pi)^{-n} \int h\left(|f|^{2}, z, w\right) b(w) d v(w)
\end{gathered}
$$

where

$$
\begin{aligned}
k(f, z, w)= & {[f(z)-f(w)] \exp \left\{-|z-w|^{2} / 4+i \operatorname{Im} \bar{w} \cdot z / 2\right\}, } \\
h\left(|f|^{2}, z, w\right)= & (2 \pi)^{-n} e^{-|z-w|^{2} / 8} \\
& \times \int|f(u)|^{2} \exp \left\{-\left|u-\left(\frac{z+w}{2}\right)\right|^{2} / 2+i \operatorname{Im}\left(\frac{z-w}{2} \cdot \bar{u}\right)\right\} d v(u) .
\end{aligned}
$$

Proof. Direct calculation.

For $f$ in $L^{\infty}\left(\mathbf{C}^{n}\right)$, we consider some properties of the convolution transform

$$
\tilde{f}(a)=(2 \pi)^{-n} \int f(z) e^{-|z-a|^{2} / 2} d v(z) .
$$

We denote by $\tilde{f}^{(m)}$ the $m$ th iterate of this transform. The map $f \rightarrow \tilde{f}$ is a smoothing operator which is clearly related to the heat equation on $\mathbf{C}^{n}=\mathbf{R}^{2 n}$. In fact,

$$
\tilde{f}(t, a)=(4 \pi t)^{-n} \int f(z) e^{-|z-a|^{2} / 4 t} d v(z)
$$

is the unique solution of the heat equation with initial values (at $t=0) f(z)$ [8]. Thus, $\tilde{f}(a)=\tilde{f}\left(\frac{1}{2}, a\right)$ is the solution of the initial value problem for $f(z)$ at $t=\frac{1}{2}$. 
We will need one estimate

LEMMA 2. For $f$ in $L^{\infty}$, we have

$$
\left|\tilde{f}^{(m)}(a)-\tilde{f}^{(m)}(b)\right| \leq 2(2 \pi)^{-1 / 2}\|f\|_{\infty} m^{-1 / 2}|a-b| .
$$

PROOF. The first step is to note that

$$
\tilde{f}(a)-\tilde{f}(b)=(2 \pi)^{-n} \int f\left(z+\frac{a+b}{2}\right)\left[e^{-|z-(a-b) / 2|^{2} / 2}-e^{-|z+(a-b) / 2|^{2} / 2}\right] d v(z) .
$$

It follows that

$$
|\tilde{f}(a)-\tilde{f}(b)| \leq(2 \pi)^{-n}\|f\|_{\infty} \int\left|e^{-|z-(a-b) / 2|^{2} / 2}-e^{-|z+(a-b) / 2|^{2} / 2}\right| d v(z) .
$$

Careful but routine analysis shows that the right-hand side of the last inequality is exactly equal to

$$
2(2 \pi)^{-1 / 2}\|f\|_{\infty} \int_{-|a-b| / 2}^{+|a-b| / 2} e^{-x^{2} / 2} d x
$$

It is immediate that

$$
|\tilde{f}(a)-\tilde{f}(b)| \leq 2(2 \pi)^{-1 / 2}\|f\|_{\infty}|a-b|
$$

Using the semigroup property of the heat kernel (or direct calculation) we see that

$$
\tilde{f}^{(m)}(a)=\tilde{f}(m / 2, a)=(2 \pi m)^{-n} \int f(z) e^{-|z-a|^{2} / 2 m} d v(z) .
$$

It follows that $\tilde{f}^{(m)}(a)=\tilde{g}(a / \sqrt{m})$ with $g(z)=f(z \sqrt{m})$. The desired result follows by applying the Lipschitz estimate above to $\tilde{g}$.

In view of the central role played by the algebra $E S V$ in our analysis, we next provide some useful examples.

THEOREM 3. The algebra ESV includes (i) $\hat{g}(z)=g(z /|z|)$ for $g$ continuous complex-valued on $S^{2 n-1}=\{z:|z|=1\}$, (ii) $f(|z|)$ for $f$ in $B C_{r} E S V$ (see [6]), (iii) $\{V+\lambda 1: \lambda \in \mathbf{C}\}$.

ProOF. (i) can be checked directly, using the uniform continuity of $g$ on $S^{2 n-1}$. We note that, for $|z-w| \leq 1$,

$$
\left|\frac{z}{|z|}-\frac{w}{|z|}\right| \leq \frac{1}{|z|}, \quad\left|\frac{w}{|z|}-\frac{w}{|w|}\right| \leq \frac{|w||| w|-| z||}{|w||z|} \leq \frac{1}{|z|}
$$

so that

$$
\left|\frac{z}{|z|}-\frac{w}{|w|}\right| \leq \frac{2}{|z|} .
$$

(ii) follows directly from the corresponding definition of $B C_{r} E S V$ [6] as the radial version of $B C E S V$ defined above.

(iii) is immediate from the definition of $E S V$.

REMARK. It follows from Theorem 3 and discussion in $[6]$ that $\exp (i \sqrt{|z|})$ is in $E S V$. On the other hand, $\exp (i \operatorname{Im}(\bar{\lambda} \cdot z))$ is not in $E S V$ unless $\lambda=0$.

The following lemma exhibits the strong interaction between $E S V$ and the transform $\tilde{f}$. 
LEMma 4. For $f$ in $E S V, f-\tilde{f}$ is in $V$.

ProOF. We write

$$
\begin{aligned}
f(a)-\tilde{f}(a) & =(2 \pi)^{-n} \int[f(a)-f(z)] e^{-|z-a|^{2} / 2} d v(z) \\
& =(2 \pi)^{-n} \int[f(a)-f(a+z)] e^{-|z|^{2} / 2} d v(z) .
\end{aligned}
$$

Thus, for $\varepsilon>0$ and $N=N(\varepsilon)$ large enough

$$
\begin{aligned}
& (2 \pi)^{-n} \int_{|z| \geq N}|f(a)-f(a+z)| e^{-|z|^{2} / 2} d v(z) \\
& \quad \leq(2 \pi)^{-n} 2\|f\|_{\infty} \int_{|z| \geq N} e^{-|z|^{2} / 2} d v(z)<\frac{\varepsilon}{2}
\end{aligned}
$$

and

$$
|f(a)-\tilde{f}(a)|<\frac{\varepsilon}{2}+(2 \pi)^{-n} \int_{|z|<N}|f(a)-f(a+z)| e^{-|z|^{2} / 2} d v(z) .
$$

Now, using the definition of $E S V$, there is an $R(\varepsilon)>0$ so that $|f(a)-f(a+z)|<\varepsilon / 2$ for $|z|<N$ whenever $|a|>R(\varepsilon)$. The desired result follows at once.

We can now establish

THEOREM 5. The following conditions are equivalent

(i) $f \in E S V$,

(ii) $f-\tilde{f} \in V$,

(iii) $f-\tilde{f}^{(m)} \in V$ for all $m \geq 1$,

(iv) $f \in \bigcap_{\varepsilon>0}(\Lambda(\varepsilon)+V)$.

Proof. ((i) $\rightarrow$ (ii)) If $f$ is in $E S V$ then $f-\tilde{f}$ is in $V$ by Lemma 4 .

((ii) $\rightarrow$ (iii)) If $f-\tilde{f} \in V$ then, by Theorem 3 and Lemma $4, f-\tilde{f}^{(2)} \in V$. Iteration and addition show that $f-\tilde{f}^{(m)} \in V$ for all $m \geq 1$.

$(($ iii $) \rightarrow($ iv $))$ Suppose $f-\tilde{f}^{(m)} \in V$ for all $m \geq 1$. By Lemma 2, $f \in$ $\bigcap_{\varepsilon>0}(\Lambda(\varepsilon)+V)$.

((iv) $\rightarrow\left(\right.$ i)) Suppose $f \in \bigcap_{\varepsilon>0}(\Lambda(\varepsilon)+V)$. Then for each $\varepsilon>0$ we have $f=g_{\varepsilon}+h_{\varepsilon}$ for $g_{\varepsilon}$ in $\Lambda(\varepsilon)$ and $h_{\varepsilon}$ in $V$. Suppose that $\left|h_{\varepsilon}(z)\right|<\varepsilon$ whenever $|z|>R(\varepsilon)$. Then

$$
\begin{aligned}
|f(a)-f(b)| & \leq\left|g_{\varepsilon / 3}(a)-g_{\varepsilon / 3}(b)\right|+\left|h_{\varepsilon / 3}(a)\right|+\left|h_{\varepsilon / 3}(b)\right| \\
& \leq(\varepsilon / 3)|a-b|+\left|h_{\varepsilon / 3}(a)\right|+\left|h_{\varepsilon / 3}(b)\right| .
\end{aligned}
$$

Thus, for $|a-b| \leq 1$ and $|a|>R(\varepsilon / 3)+1$, we have $|b|>R(\varepsilon / 3)$ and $\left|h_{\varepsilon / 3}(a)\right|<\varepsilon / 3$, $\left|h_{\varepsilon / 3}(b)\right|<\varepsilon / 3$ so $|f(a)-f(b)|<\varepsilon$. Thus, $f$ is in $E S V$.

COROLLARY. The following conditions are equivalent:

(i) $f$ is in $B C E S V$,

(ii) $f-\tilde{f} \in C_{0}$,

(iii) $f-\tilde{f}^{(m)} \in C_{0}$ for all $m \geq 1$,

(iv) $f \in \bigcap_{\varepsilon>0}\left(\Lambda(\varepsilon)+C_{0}\right)$.

Proof. Clear.

REMARK. Theorem 5 implies that the class $E S V$ has some significance in the classical analysis of the initial value problem for the heat equation. 
3. The symbol calculus for Toeplitz operators. We begin with a discussion of the Berezin symbol $[\mathbf{3}]$ and a related averaging operation over a representation of the Heisenberg group. This averaging operation appears to be of some independent interest and is extremely useful in our subsequent analysis.

On $H^{2}(d \mu)$, we have the unitary operator-valued map

$$
a \rightarrow W_{a}=e^{i T_{\operatorname{Im}(\bar{a} \cdot z)}}=T_{\exp \left\{|a|^{2} / 4+i \operatorname{Im}(\bar{a} \cdot z)\right\}}
$$

for $a$ in $\mathbf{C}^{n}$ (see [6]). The map $a \rightarrow W_{a}$ extends to a map from $\mathbf{C}^{n}$ to unitary operators acting on $L^{2}(d \mu)$ by the formula $[\mathbf{1}, 6] W_{a}=k_{a}(z) t_{a}$ where $k_{a}(z)=$ $\exp \left\{\bar{a} \cdot z / 2-|a|^{2} / 4\right\}$ and $\left(t_{a} f\right)(z)=f(z-a)$ for $f$ in $L^{2}(d \mu)$. For $e_{a}(z)=$ $\exp \{i \operatorname{Im}(\bar{a} \cdot z)\}$, we also consider the unitary operator-valued map $a \rightarrow M_{e_{a}}$ on $L^{2}(d \mu)$.

It is not hard to check that both $a \rightarrow W_{a}$ and $a \rightarrow M_{e_{a}}$ are weakly continuous on $L^{2}(d \mu)$. Using the identities

$$
W_{a} W_{b}=e_{a}(b / 2) W_{a+b}, \quad M_{e_{a}} M_{e_{b}}=M_{e_{a+b}},
$$

it follows that $a \rightarrow W_{a}, a \rightarrow W_{a}^{*}, a \rightarrow M_{e_{a}}, a \rightarrow M_{e_{a}}^{*}$ are all strongly continuous maps.

For $\alpha, \beta$ complex numbers of modulus one, we now have representations of the Heisenberg group on $L^{2}(d \mu)$ and $H^{2}(d \mu)$ via $(\alpha, a) \rightarrow \alpha W_{a}$ and the foregoing identities. Note that the multiplication law for the Heisenberg group is just

$$
(\alpha, a) \cdot(\beta, b)=\left(\alpha \beta e_{a}(b / 2), a+b\right) .
$$

Of course, as is well known [7], the representation on $L^{2}(d \mu)$ is reducible while the representation on $H^{2}(d \mu)$ is irreducible.

For $A$ a bounded operator on $L^{2}(d \mu)$ or $H^{2}(d \mu)$, we can now define an averaging operation by

$$
\hat{A}=\int W_{a}^{*} A W_{a} d \mu(a) .
$$

Note that the integrand is strongly continuous in $a$ and uniformly bounded for each fixed $A$. For a discussion of such integrals, see [7]. We note that $\hat{A}$ is determined by

$$
\langle\hat{A} f, g\rangle=\int\left\langle W_{a}^{*} A W_{a} f, g\right\rangle d \mu(a) .
$$

Recall that we defined

$$
\tilde{f}(\lambda)=(2 \pi)^{-n} \int f(z) e^{-|z-\lambda|^{2} / 2} d v(z) .
$$

On $H^{2}(d \mu)$ we have the Berezin symbol [3]

$$
\tilde{A}(\lambda)=\left\langle A k_{\lambda}, k_{\lambda}\right\rangle
$$

for any bounded operator $A$. It was shown in $[\mathbf{3}]$ that $\tilde{A}(\lambda)$ is always a bounded smooth function which $A$ determines uniquely (for any polynomials $p, q,\langle A p, q\rangle$ is obtained by evaluating appropriate derivatives of $\tilde{A}(\lambda)$ at $\lambda=0)$. Since $\left\{k_{\lambda}\right\}$ converges weakly to 0 as $|\lambda| \rightarrow \infty$, it is easy to see that for $A$ in $\mathcal{K}$ (a compact operator) $\tilde{A}(\lambda)$ is in $C_{0}$. It is easy to check $[\mathbf{3}]$ that $\tilde{T}_{f}=\tilde{f}$.

The relation between $\hat{A}$ and $\tilde{A}$ can now be determined. 
THEOREM 6. We have $\hat{A}=T_{\tilde{A}}$ for all bounded operators $A$ on $H^{2}(d \mu)$. The map $A \rightarrow \hat{A}$ is a 1-1 norm-decreasing positive linear map from all bounded operators to Toeplitz operators with symbols in $B C$. We have $\hat{T}_{f}=T_{\tilde{f}}$ on $H^{2}(d \mu)$ while, on $L^{2}(d \mu), \hat{M}_{f}=M_{\tilde{f}}$ and $\widehat{A P}=\hat{A} P, \widehat{P A}=P \hat{A}$.

ProOF. By direct calculation $W_{a} k_{\lambda}=e_{a}(\lambda / 2) k_{\lambda+a}$ so $\tilde{\hat{A}}=\tilde{T}_{\tilde{A}}$ and $\hat{A}=T_{\tilde{A}}$. That $A \rightarrow \hat{A}$ is 1-1 follows from the unicity of $\tilde{A}$ and the fact that the symbol of a Toeplitz operator uniquely determines the operator $\left(T_{f}=0\right.$ if and only if $f=0$ [6]). The remaining observations are checked easily.

REMARK. Theorem 6 shows that $A \rightarrow \hat{A}$ is almost a conditional expectation from all bounded operators to Toeplitz operators with $B C$ symbols. Using $\hat{T}_{f}=T_{\tilde{f}}$, it is clear that repeated application of ^ increasingly smooths the symbol by Lemma 2. This property of ` will be used in what follows and should have other applications. The well-known irreducibility of the $\left\{W_{a}: a \in \mathbf{C}^{n}\right\}$ on $H^{2}(d \mu)$ follows easily from Theorem 6 since $\left[A, W_{a}\right]=0$ for all $a$ implies $A=\hat{A}=T_{\tilde{A}}$ so $\tilde{A}=\tilde{A}^{(2)}$ and, by iteration, $\tilde{A}=\tilde{A}^{(m)}$. Lemma 2 then implies that $\tilde{A}(\lambda)$ is a constant function so that $A$ is a scalar multiple of $I$.

Using the Berezin symbol, it is easy to show

THEOREM 7. For $f$ in $B, \tilde{f}$ is in $C_{0}$.

Proof. Recall that $\left\langle T_{f} k_{\lambda}, k_{\lambda}\right\rangle=\tilde{T}_{f}(\lambda)=\tilde{f}(\lambda)$. Now $k_{\lambda} \rightarrow 0$ (weakly) as $|\lambda| \rightarrow \infty$ so compactness of $T_{f}$ implies that $T_{f} k_{\lambda} \rightarrow 0$ (strongly) and so $\tilde{f} \in C_{0}$.

We also have

THEOREM 8. $E S V \cap B=V$.

PROOF. It is a direct calculation in [6] that for $f$ in $L^{\infty}$ with compact support, $f \in B$. It follows from the fact that $B$ is closed that $V \subset B$ and, hence, $V \subset$ $E S V \cap B$.

For the converse, suppose $f \in E S V \cap B$. By Lemma $4, f-\tilde{f} \in V$ while Theorem 7 implies $\tilde{f} \in C_{0}$. It follows immediately that $f \in V$.

We also have

LEMMA 9. $V \subset Q \cap B$.

ProOF. By a direct operator-theoretic argument

$$
\Gamma \cap B=\left\{f:|f|^{2} \in B\right\}=Q \cap B .
$$

Moreover, $f \in V$ if and only if $|f|^{2} \in V$. By Theorem 8, $V \subset B$ so, for $f$ in $V,|f|^{2}$ is in $B$ and $f$ is in $Q \cap B$.

We will need

LEMMA 10. $f \in Q$ if and only if $\left[M_{f}, P\right]$ is compact.

Proof. If $\left[M_{f}, P\right]$ is compact then $(I-P) M_{f} P$ and $(I-P) M_{\bar{f}} P$ are also compact so $f$ is in $\Gamma \cap \bar{\Gamma}=Q$.

For the converse, note that for $f$ in $Q$ we have $(I-P) M_{f} P$ and $(I-P) M_{\bar{f}} P$ compact. Hence, $P M_{f}(I-P)$ is compact so

$$
(I-P) M_{f} P-P M_{f}(I-P)=\left[M_{f}, P\right]
$$

is compact. 
Recall that $U$ is the unitary transformation from $L^{2}(d \mu)$ onto $L^{2}\left((2 \pi)^{-n} d v\right)$ given by

$$
(U g)(z)=e^{-|z|^{2} / 4} g(z) .
$$

As customary, $K$ denotes the ideal of compact operators.

THEOREM 11. ESV $\subset Q$.

Proof. If $f$ is in $E S V$ then, by Theorem $5, f=g_{\varepsilon}+h_{\varepsilon}$ with $g_{\varepsilon}$ in $\Lambda(\varepsilon)$ and $h_{\varepsilon}$ in $V$. By Proposition 1,

$$
U\left[M_{g_{\varepsilon}}, P\right] U^{*} b(z)=(2 \pi)^{-n} \int k\left(g_{\varepsilon}, z, w\right) b(w) d v(w)
$$

and

$$
\left|k\left(g_{\varepsilon}, z, w\right)\right| \leq \varepsilon|z-w| \exp \left\{-|z-w|^{2} / 4\right\}
$$

so

$$
\left|U\left[M_{g_{\varepsilon}}, P\right] U^{*} b(z)\right| \leq \varepsilon(2 \pi)^{-n} \int|z-w| e^{-|z-w|^{2} / 4}|b(w)| d v(w) .
$$

Let

$$
(B b)(z)=(2 \pi)^{-n} \int e^{-|z-w|^{2} / 4}|z-w| b(w) d v(w) .
$$

Then $B$ is a bounded convolution operator. In fact,

$$
\|B\|=(2 \pi)^{-n} \int e^{-|w|^{2} / 4}|w| d v(w) .
$$

It follows that

$$
\left\|\left[M_{g_{\varepsilon}}, P\right]\right\| \leq \varepsilon\|B\| .
$$

Recall that, by Lemma $9, M_{h_{\varepsilon}} P$ and $P M_{h_{\varepsilon}}$ are compact operators. It follows that

$$
\left\|\left[M_{f}, P\right]+\mathcal{K}\right\| \leq \varepsilon\|B\|
$$

and, since $\varepsilon>0$ is arbitrary, that $\left[M_{f}, P\right]$ is compact. An application of Lemma 10 completes the proof.

REMARK. It should be pointed out that Theorem 11 can also be obtained as an application of results in $[\mathbf{1 1}]$.

Suppose that $X$ is a Borel space with $\nu$ a positive measure on $X$ and $\nu(X)$ finite. Suppose further that $A(x)$ is a weakly measurable function on $X$ with range contained in the bounded operators on a separable Hilbert space $H$. Recall that $\int A(x) d v(x)=A$ is a bounded operator on $H$ defined, for $f, g$ in $H$, by

$$
\langle A f, g\rangle \equiv \int\langle A(x) f, g\rangle d \nu(x) .
$$

The next lemma is essential for our analysis. We thank William Zame for this simplified variant of our original version. 
LEMMA 12. If $\|A(x)\| \leq M$ and $A(x)$ is a compact operator for all $x$ in $X$ then $\int A(x) d \nu(x)$ is also compact.

PROOF. For $\left\langle e_{k}: k=1,2,3, \ldots\right\rangle$ an orthonormal basis for $H$, write $P_{k}$ for the orthogonal projection operator with range spanned by $\left\langle e_{1}, e_{2}, \ldots, e_{k}\right\rangle$. Clearly, $P_{k} A(x) P_{k}-A(x)$ is weakly measurable. Given $\varepsilon>0$,

$$
E_{k}=\left\{x \in X:\left\|P_{k} A(x) P_{k}-A(x)\right\|<\varepsilon\right\}
$$

is a measurable set since

$$
\left\|P_{k} A(x) P_{k}-A(x)\right\|=\sup _{f, g \in D}\left\langle\left[P_{k} A(x) P_{k}-A(x)\right] f, g\right\rangle
$$

for $D$ a dense countable subset of the unit ball of $H$.

Note that $\bigcup_{k \geq 1} E_{k}=X$ since $A(x)$ is compact for all $x$ in $X$. We define

$$
E_{k}^{\prime}=E_{k} \backslash \bigcup_{j=1}^{k-1} E_{j}^{\prime}, \quad E_{1}^{\prime}=E_{1}
$$

so that the $E_{k}^{\prime}$ are measurable and disjoint with

$$
\bigcup_{k \geq 1} E_{k}=\bigcup_{k \geq 1} E_{k}^{\prime}=X .
$$

Since $\nu(X)$ is finite, there is an $m$ so that $\sum_{k>m} \nu\left(E_{k}^{\prime}\right)<\varepsilon / M$. We now have

$$
\begin{aligned}
\int_{X} A(x) d \nu(x)= & \sum_{k=1}^{m} \int_{E_{k}^{\prime}} P_{k} A(x) P_{k} d \nu(x) \\
& +\sum_{k=1}^{m} \int_{E_{k}^{\prime}}\left[A(x)-P_{k} A(x) P_{k}\right] d \nu(x) \\
& +\int_{\cup_{k>m} E_{k}^{\prime}} A(x) d \nu(x) .
\end{aligned}
$$

The last two terms on the right have norms less than $\varepsilon \nu(X)$ and $\varepsilon$ respectively while the first term has range contained in the range of $P_{m}$. Since $\varepsilon>0$ was arbitrary, the desired result follows immediately.

THEOREM 13. We have $\Gamma \subset E S V+Q \cap B$.

PROOF. Note that

$$
Q \cap B=\left\{f:|f|^{2} \in B\right\}=\Gamma \cap B .
$$

We will show, for $f$ in $\Gamma$, that $f-\tilde{f}$ is in $Q \cap B$ and that $\tilde{f}$ is in $E S V$.

Since $T_{\tilde{f}}=\hat{T}_{f}$ by Theorem 6 , it follows that

$$
T_{f-\tilde{f}}=\int\left[T_{f}, W_{a}^{*}\right] W_{a} P d \mu(a)
$$

on $H^{2}(d \mu)$. Writing $H_{f}=(I-P) M_{f} P$ and letting

$$
d \tilde{\mu}(a)=e^{|a|^{2} / 4} d \mu(a),
$$


direct calculation shows that

$$
\begin{aligned}
T_{f-\tilde{f}}= & \int P M_{e_{-a}} H_{f} W_{a} P d \tilde{\mu}(a) \\
& -H_{\tilde{f}}^{*} \int M_{e_{-a}} P W_{a} P d \tilde{\mu}(a) .
\end{aligned}
$$

The fact that $d \tilde{\mu}$ has finite total mass and Lemma 12 together with the fact that $H_{f}$ is compact for $f$ in $\Gamma$ allow us to conclude that the first integral is a compact operator.

Next, we compute

$$
\begin{aligned}
J & =\int M_{e_{-a}} P W_{a} d \tilde{\mu}(a) \\
& =\int M_{e_{-a}} P k_{a}(z) t_{a} P d \tilde{\mu}(a) \\
& =\int e_{-a} e^{\bar{a} \cdot z / 2} t_{a} P d \mu(a) \\
& =\int e^{a \cdot \bar{z} / 2} t_{a} P d \mu(a)=P .
\end{aligned}
$$

The last step uses Fubini's theorem and the fact that the $d \mu(a)$ integral of an analytic function of $a$ is just the constant term in the McLaurin expansion. It follows that

$$
H_{\bar{f}}^{*} \int M_{e_{-a}} P W_{a} P d \tilde{\mu}(a)=H_{\bar{f}}^{*} J=P M_{f}(I-P) P=0
$$

so the second integral in the expression for $T_{f-\tilde{f}}$ is zero and $T_{f-\tilde{f}}$ is compact. Hence $f-\tilde{f}$ is in $B$.

Next, using the fact that $\left[W_{a}, P\right]=0$ on $L^{2}(d \mu)$ and Theorem 6 , it is not hard to see for $H_{f}=(I-P) M_{f} P$ that $\hat{H}_{f}=H_{\tilde{f}}$. It follows from Lemma 12, that for $f$ in $\Gamma$, since $H_{f}$ is compact, $\hat{H}_{f}$ must be compact and so $\tilde{f}$ is in $\Gamma$. Thus, $f-\tilde{f}$ is in $\Gamma \cap B=Q \cap B$.

Since $f-\tilde{f}$ is in $B, \tilde{f}-\tilde{f}^{(2)}$ is in $C_{0}$ by Theorem 7 . It follows immediately from Theorem 5 that $\tilde{f}$ is in $E S V$.

Finally, we have the characterization

THEOREM B. $\Gamma=E S V+Q \cap B=Q$.

Proof. Combining Theorems 11 and 13 we see that $\Gamma \subset E S V+Q \cap B \subset Q$. But $Q=\Gamma \cap \bar{\Gamma} \subset \Gamma$ so the inclusions above must be equalities.

Next, we establish a useful relation between $|f(z)|^{2}$ and $\widetilde{|f|^{2}}(a)$ where, as earlier

$$
\tilde{f}(a)=(2 \pi)^{-n} \int f(z) e^{-|z-a|^{2} / 2} d v(z) .
$$

We have

LEMMA 14. $\left\|\widetilde{|f|^{2}}\right\|_{\infty} \leq\left\|P M_{|f|^{2}} P\right\| \leq 4^{n}\left\|\widetilde{|f|^{2}}\right\|_{\infty}$.

PROOF. Note first that for $k_{\lambda}(z)=e^{\bar{\lambda} \cdot z / 2-|\lambda|^{2} / 4}$ we have

$$
\left\langle P M_{|f|^{2}} P k_{\lambda}, k_{\lambda}\right\rangle=\widetilde{|f|^{2}}(\lambda) \text {. }
$$


Since $\left\|k_{\lambda}\right\|_{2}=1$, it follows that

$$
\left\|P M_{|f|^{2}} P\right\| \geq\left\|\widetilde{|f|^{2}}\right\|_{\infty}
$$

The remaining estimate is more subtle. Using Proposition 1, we find that for $b$ in $L^{2}\left((2 \pi)^{-n} d v\right)$

$$
U P M_{|f|^{2}} P U^{*} b(z)=(2 \pi)^{-n} \int h\left(|f|^{2}, z, w\right) b(w) d v(w)
$$

where

$$
\begin{aligned}
\left|h\left(|f|^{2}, z, w\right)\right| & \leq(2 \pi)^{-n} e^{-|z-w|^{2} / 8} \int|f(u)|^{2} e^{-|u-(z+w) / 2|^{2} / 2} d v(u) \\
& \leq e^{-|z-w|^{2} / 8}\left\|\widetilde{\left.f\right|^{2}}\right\|_{\infty}
\end{aligned}
$$

It follows that

$$
\begin{aligned}
\left|U P M_{|f|^{2}} P U^{*} b(z)\right| & \leq\left\|\widetilde{|f|^{2}}\right\|_{\infty}(2 \pi)^{-n} \int e^{-|z-w|^{2} / 8}|b(w)| d v(w) \\
& \leq\left\|\widetilde{|f|^{2}}\right\|_{\infty}(A|b|)(z)
\end{aligned}
$$

where

$$
(A b)(z)=(2 \pi)^{-n} \int e^{-|z-w|^{2} / 8} b(w) d v(w)
$$

Thus we have

$$
\left\|U P M_{|f|^{2}} P U^{*} b\right\|_{2} \leq\|A\|\left\|\widetilde{|f|^{2}}\right\|_{\infty}\|b\|_{2}
$$

and so

$$
\left\|P M_{|f|^{2}} P\right\| \leq\|A\|\left\|\widetilde{|f|^{2}}\right\|_{\infty}
$$

An easy computation shows that the convolution operator $A$ has $\|A\|=4^{n}$ and the desired estimate follows.

REMARK. Using the lemma above, it is not hard to check that $\left\|M_{f} P\right\|$ is finite if and only if $\widetilde{|f|^{2}}$ is bounded even if $f$ is not in $L^{\infty}$. We will return to this point in the last section of this paper.

We can now give a complete characterization of $Q \cap B$.

TheOREM C. $Q \cap B=\left\{f \in L^{\infty}: \widetilde{|f|^{2}} \in C_{0}\right\}$.

Proof. If $f$ is in $Q \cap B$ then $M_{f} P$ is compact so $\left(M_{f} P\right)^{*}\left(M_{f} P\right)=P M_{|f|^{2}} P$ is compact and $\left\langle P M_{|f|^{2}} P k_{\lambda}, k_{\lambda}\right\rangle=\widetilde{|f|^{2}}(\lambda)$ is in $C_{0}$.

For the converse, suppose $\widetilde{|f|^{2}}$ is in $C_{0}$. By Lemma $9, V \subset Q \cap B$. Let $\chi_{\rho}$ be the characteristic function of $\{z:|z|>\rho\}$. Then

$$
M_{f} P=M_{f_{\chi_{\rho}}} P+M_{f\left(1-\chi_{\rho}\right)} P
$$

and $f\left(1-\chi_{\rho}\right)$ is in $V$ so $M_{f\left(1-\chi_{\rho}\right)} P$ is compact. Hence, for $M_{f} P$ to be compact (and $f$ to be in $Q \cap B$ ) it suffices to show that $\operatorname{Lim}_{\rho \rightarrow \infty}\left\|M_{f_{\chi_{\rho}}} P\right\|=0$. Using Lemma 14 , it is enough to check for $g_{\rho}(\lambda)=\widetilde{\chi_{\rho}|f|^{2}}(\lambda)$ that $\operatorname{Lim}_{\rho \rightarrow \infty}\left\|g_{\rho}\right\|_{\infty}=0$. Note that the functions $g_{\rho}$ are in $C_{0}$ since $\widetilde{|f|^{2}}$ is in $C_{0}$. Moreover, the $g_{\rho}$ are nonnegative 
with $g_{\rho^{\prime}}(a) \leq g_{\rho}(a)$ whenever $\rho^{\prime}>\rho$ and $\operatorname{Lim}_{\rho \rightarrow \infty} g_{\rho}(a)=0$ for each $a$ in $\mathbf{C}^{n}$. An elementary variant of Dini's Theorem completes the proof.

We have seen that $V$ is in $Q \cap B$ while, for $f$ in $Q \cap B, \widetilde{|f|^{2}} \in C_{0}$. This raises the question of whether $Q \cap B$ is larger than $V$. The anser is "yes" by a construction which exhibits a somewhat surprising property of the heat equation.

EXAMPLE. On $\mathbf{C}$, let $D_{j}$ be the open unit disc of radius $j^{-1}$ centered at $j$ for $j=1,2,3, \ldots$ along the real axis. Let $N=\bigcup_{j} D_{j}$ and let $f$ be a continuous nonnegative real-valued function on $\mathbf{C}$ with $0 \leq f(z) \leq 1, f(j)=1(j=1,2,3, \ldots)$ and support $(f)$ contained in $N$. Then $f$ is clearly not in $V$ but $\tilde{f}$ and $\widetilde{f^{2}}$ are in $C_{0}$ by straightforward estimates.

Finally, we have

THEOREM D. For $A$ a bounded operator on $H^{2}(d \mu),\left[A, W_{a}\right]$ is compact for all $a$ in $\mathbf{C}^{n}$ if and only if $A-T_{f}$ is compact for some $f$ in $E S V$.

ProOF. If $A-T_{f}$ is compact for $f$ in $E S V$ then Proposition A, Theorem 11, and the fact that $W_{a}$ is a Toeplitz operator (discussed earlier) imply that $\left[A, W_{a}\right]$ is compact for all $a$.

For the converse, note by Theorem $6, \hat{A}=T_{f}$ with $f=\tilde{A}(\lambda)$. Thus, we have

$$
A-T_{f}=A-\hat{A}=\int\left(A-W_{a}^{*} A W_{a}\right) d \mu(a)=\int\left[A, W_{a}^{*}\right] W_{a} d \mu(a) .
$$

By Lemma 12 and the fact that $W_{a}^{*}=W_{-a}$, we see that the last integral is a compact operator. It follows that $\tilde{A}-\tilde{T}_{f}=f-\tilde{f}$ is in $V$. It follows from Theorem 5 that $f$ is in $E S V$.

4. The algebra $\tau(Q)$. We now use the analysis of $\S 3$ to determine the structure of $\tau(Q)$. We first identify some function algebra relations which are implicit in $\S 3$.

THEOREM 15. There are $C^{*}$-algebra isomorphisms

$$
Q / Q \cap B \simeq E S V / V \simeq B C E S V / C_{0} .
$$

Proof. Direct consequence of Theorem B $(Q=E S V+Q \cap B)$ and Theorem $8(E S V \cap B=V)$. We also use the fact that $f-\tilde{f}$ is in $V$ for $f$ in $E S V$ so that $E S V=B C E S V+V$.

We use the standard notation of $\tau(X)$ for the $C^{*}$-algebra generated by $\left\{T_{f}: f \in\right.$ $X\}$. We now have

THEOREM 16. $\tau(Q)$ contains $\mathcal{K}$ and the map $\psi(f)=\pi\left(T_{f}\right)$ induces a *-isomorphism between $B C E S V / C_{0}$ and $\tau(Q) / K$.

Proof. Note that for $\chi_{\rho}$ the characteristic function of $\{z:|z|>\rho\}$ we have, as $\rho \rightarrow \infty, T_{f\left(1-\chi_{\rho}\right)} \rightarrow T_{f}$ weakly for all $f$ in $L^{\infty}$. Since $f\left(1-\chi_{\rho}\right)$ is in $V$ it follows that $\tau\left(L^{\infty}\right)$ is contained in the weak closure of $\tau(V)$. But $\tau\left(L^{\infty}\right)$ contains $\left\{W_{a}\right.$ : a in $\left.\mathbf{C}^{n}\right\}$ (see $\S 1$ ) and this set is irreducible by an earlier remark. It follows that $\tau\left(L^{\infty}\right), \tau(Q)$, and $\tau(V)$ are also irreducible. Since $\tau(V) \subset \mathcal{K}$, it follows from standard $C^{*}$-algebra results that $K=\tau(V)$.

Next, using Proposition A and Theorems 13 and 15, we see that $\tau(Q)$ is the closure of

$$
S=\left\{T_{f}+K: f \in B C E S V, K \in \mathcal{K}\right\}
$$


For $f$ in $B C E S V$, let $\psi(f)=\pi\left(T_{f}\right)$. Then $\psi$ is a $*$-homomorphism from $B C E S V$ onto $\tau(Q) / K$ since the image of any *-homomorphism is closed. It follows that $\tau(Q)=S$ and that

$$
B C E S V / \operatorname{ker} \psi \simeq \tau(Q) / K .
$$

Finally, we note that, by Theorem 8 ,

$$
\begin{aligned}
\operatorname{ker} \psi & =\left\{f \in B C E S V: T_{f} \in \mathcal{K}\right\}=B C E S V \cap B \\
& =B C \cap E S V \cap B=B C \cap V=C_{0} .
\end{aligned}
$$

Combining Theorems 15 and 16, we have

THEOREM E. $\pi\{\tau(Q)\} \simeq Q / Q \cap B \simeq E S V / V \simeq B C E S V / C_{0}$.

In the rest of this section, we consider the Fredholm theory and index problem for $\tau(Q)$. The following lemma and Theorem 18 appear in [12].

LEMMA 17. For $f$ in BCESV, the following conditions are equivalent: there is a $g$ in $B C E S V$ with $g f-1$ in $C_{0}$, and (ii) for some $R>0$ there is an $m>0$ with $|f(z)| \geq m$ for all $z$ with $|z| \geq R$.

ProOF. (i) $\rightarrow$ (ii). Suppose $g f-1=h \in C_{0}$. If there is a sequence $\left\{z_{k}\right\}$ with $\left|z_{k}\right| \rightarrow \infty$ and $\left|f\left(z_{k}\right)\right|<\varepsilon$ for each $\varepsilon>0$, then

$$
\left|1+h\left(z_{k}\right)\right|=\left|g\left(z_{k}\right)\right|\left|f\left(z_{k}\right)\right| \leq \varepsilon\|g\|_{\infty}
$$

and, for $\varepsilon$ small and $k$ large, we have a contradiction.

(ii) $\rightarrow$ (i). There are two cases depending on the dimension of $\mathbf{C}^{n}$.

CASE 1. $n=1$. If $f(z)$ has winding number $r$ on $|z|=R$ then the function $f(z)(\bar{z} /|z|)^{r}$ on $|z| \geq R$ extends to $F(z)$ on $\mathbf{C}^{1}$ with $|F(z)| \geq m^{\prime}>0$ and $F$ in $B C E S V$ by a standard homotopy argument. It follows that $1 / F$ is in $B C E S V$ and $(1 / F) f=(z /|z|)^{r}$ for $|z| \geq R$. Let

$$
G(z)= \begin{cases}(\bar{z} /|z|)^{r}, & |z| \geq R, \\ (\bar{z} / R)^{r}, & |z|<R .\end{cases}
$$

Then $(G / F) f-1=0$ for $|z| \geq R$ and $g=G / F$ will do.

CASE 2. $n>1$. Here, $f(z)$ on $|z| \geq R$ extends to a continuous $F(z)$ on $\mathbf{C}^{n}$ with $|F(z)| \geq m^{\prime}>0$ and $F(z)$ in $B C E S V$ by a standard homotopy argument. It follows that $1 / F$ is in $B C E S V$ and, for $g=1 / F, g f-1=0$ on $|z| \geq R$.

Now let $\sigma(x)$ denote the spectrum of $x$ for $x$ in any Banach algebra with identity. We will be concerned with the abelian $C^{*}$-algebra $B C E S V / C_{0}$. have

THEOREM 18. For $f$ in BCESV and $[f]$ the class of $f$ in $B C E S V / C_{0}$, we

$$
\sigma([f])=\bigcap_{R>0} \operatorname{closure}[f(z:|z| \geq R)]
$$

PROOF. Recall that $\lambda$ is in $\sigma([f])$ if and only if there is no $[g]$ in $B C E S V / C_{0}$ with $[g][f-\lambda 1]=[1]$, or, equivalently, if and only if there is no $g$ in $B C E S V$ with $g(f-\lambda 1)-1 \in C_{0}$. The desired result follows immediately from Lemma 17 .

COROLlaRY 1. $\sigma([f])$ is connected for all $[f]$ in $B C E S V / C_{0}$.

PROOF. By Theorem $18, \sigma([f])$ is the intersection of a nested family of compact connected sets and is, therefore, connected. 
Let $\mathcal{M}$ be the maximal ideal space of $B C E S V / C_{0}$. We now have

Corollary 2. $\mathcal{M}$ is connected.

PROOF. If not, by standard facts, $B C E S V / C_{0}$ would have a nontrivial idempotent element with spectrum $\{0,1\}$. This is impossible by Corollary 1 .

Recall that $\sigma_{e}(A) \equiv \sigma[\pi(A)]$ for $\pi$ the quotient map from $B(H)$ onto $B(H) / K$.

COROllary 3. For $f$ in $Q, \sigma_{e}\left(T_{f}\right)$ is connected.

PROOF. Easy from Corollary 1 and Theorem 16.

REMARK. Since $C C R\left(\mathbf{C}^{n}\right)$ contains nontrivial projections $[\mathbf{1 3}], \sigma_{e}\left(T_{f}\right)$ must be disconnected for some trigonometric polynomials $f$.

Finally, we can establish an index theorem for $\tau(Q)$ along familiar lines. Using the characterization of $S=\tau(Q)$ in the proof of Theorem 16, it suffices to consider $T_{f}$ for $f$ in $B C E S V$.

THEOREM 19. For $f$ in BCESV, $T_{f}$ is Fredholm if and only if $|f(z)| \geq m>0$ for all $z$ with $|z| \geq R$ for some $R$. For such $f$, index $\left(T_{f}\right)=-$ winding number $\left(\left.f\right|_{|z|=R}\right)$ when $n=1$ and index $\left(T_{f}\right)=0$ for $n>1$.

PrOOF. It is easy to check that $T_{f}$ is Fredholm if and only if $\left[T_{f}\right]$ is invertible in $\tau(Q) / K$. By Lemma 17 and Theorem 16 this is true if and only if $|f(z)| \geq m>0$ for all $z$ with $|z| \geq R$ for some $R$.

Suppose $f$ in $B C E S V$ satisfies $|f(z)| \geq m>0$ for all $Z$ with $|z| \geq R$. For $n \geq 2$, as noted before $f-g$ is in $C_{0}$ for some $g$ in $B C E S V$ with $|g(z)| \geq m^{\prime}>0$ for all $z$. For $n=1$, there is an integer $r$ and there is a $g$ in $B C E S V$ with $|g(z)| \geq m^{\prime}>0$ for all $z$ so that $(\bar{z} /|z|)^{r} f(z)-g(z)$ is in $V$. Moreover, $g$ has winding number zero around any circle in C. An easy calculation in [6] shows that index $T_{(\bar{z} /|z|)^{r}}=r$, which is the winding number of $f$ around $|z|=R$. The previous discussion of $\tau(Q)$ and standard Fredholm theory show that $r+\operatorname{index} T_{f}=\operatorname{index} T_{g}$ for $n=1$ while index $T_{f}=\operatorname{index} T_{g}$ for $n \geq 2$. Thus, it will suffice to check that index $T_{g}=0$.

Since $|g|$ is bounded below and $g$ is in $B C E S V, 1 /|g|$ is also in $B C E S V$ and, for $G=g /|g|$

$$
\text { index } T_{G}=\text { index } T_{g}+\text { index } T_{1 /|g|} \text {. }
$$

Since $t(1 /|g|)+(1-t) 1$ is an arc of invertible elements in $B C E S V$, index $T_{1 /|g|}=0$ and we need only check that index $T_{G}=0$ for $G$ in $B C E S V$ with $|G|=1$.

By monodromy, $G$ has a continuous argument $F$ on $\mathbf{C}^{n}$ (of course, $F$ need not be bounded) so $G(z)=\exp \{i F(z)\}$. We can check that $F(z)$ is an $E S V$-like function in the sense that

$$
\operatorname{Lim}_{\rho \rightarrow \infty} \sup _{\substack{|z| \geq \rho \\|w-z| \leq 1}}|F(z)-F(w)|=0 .
$$

Choose $\delta(\varepsilon)$ so that $\left|e^{i a}-1\right|<\delta(\varepsilon)$ and $|a| \leq 1$ implies $|a|<\varepsilon$. For $\varepsilon>0$ given, consider a fixed $z$ with $|z|$ large enough that $|G(z)-G(w)|<\delta(\varepsilon)$ for $|w-z| \leq 1$. It follows that for all such $w$, there is an integer-valued function $k(w)$ with $|F(z)-F(w)-2 \pi k(w)|<\varepsilon$. By continuity of $F, k(z)=0$ and $k(\cdot)$ must be constant so $|F(z)-F(w)|<\varepsilon$. It follows that, for any integer $m>0$, $H_{m}=\exp \{i F / m\}$ is in $B C E S V$ with $\left(H_{m}\right)^{m}=G$. Hence,

$$
\text { index } T_{G}=\operatorname{index} T_{H_{m}}^{m}=m \text { index } T_{H_{m}}
$$

and so index $T_{G}=0$. 
EXAMPLE. Consider the function on $\mathbf{C}$

$$
f(z)= \begin{cases}z, & |z|<1 \\ z /|z|, & |z| \geq 1 .\end{cases}
$$

It is easy to check that $f$ is in $B C E S V$ and $T_{f}$ is Fredholm with index $\left(T_{f}\right)=-1$.

5. Extensions and generalizations. In this section, we discuss some extensions and possible generalizations of our results.

We note, first, that Theorems B and C hold when $L^{\infty}$ is replaced by the function space

$$
L=\left\{f \text { measurable: } \widetilde{|f|^{2}} \in B C\right\}
$$

in the definition of $\Gamma, B, Q$. For $f$ in $L$, Lemma 14 shows that $T_{f}$ is a bounded operator. As for $f$ in $L^{\infty}, f=\tilde{f}+(f-\tilde{f})$ gives a decomposition of $Q$ as $E S V+Q \cap B$. Note that $E S V$ can be defined as before: the unbounded part of $f$ is absorbed in $Q \cap B$.

Analogs of our results are likely to hold for the classical domains. In particular, the Bergman space of the unit disc $D$, in $\mathbf{C}^{\mathbf{1}}$, has been profitably studied. The group $S L(2, \mathbf{R})$ acts on $H^{2}(D, d A)$ by linear fractional transformations and this group plays a role like that of the Heisenberg group on $H^{2}\left(\mathbf{C}^{n}, d \mu\right)$. K. H. Zhu has recently obtained a characterization of the algebra $Q$ on $H^{2}(D, d A)$ in terms of oscillation near the boundary [16]. The following result is useful in the analysis of $Q$ on general classical domains.

THEOREM 20. Let $\Omega$ be a bounded Cartan domain in $\mathbf{C}^{n}$ with $d v$ the usual volume measure. Suppose $P$ is the usual orthogonal projection from $L^{2}(\Omega, d v)$ onto the Bergman subspace of holomorphic functions, $H^{2}(\Omega, d v)$. Then $\left.P\right|_{L^{\infty}(\Omega)}$ is a compact operator from the Banach space $L^{\infty}(\Omega)$ to $H^{2}(\Omega, d v)$.

Proof. Let $E$ be the injection of $L^{\infty}(\Omega)$ into $L^{2}(\Omega, d v)$. Then $\left.P\right|_{L^{\infty}(\Omega)}=P E$ and, for $M_{\chi_{\sigma}}$ the operator of multiplication by the characteristic function of the compact set $\sigma, \sigma \subset \Omega$, we have

$$
P E=P M_{\chi_{\sigma}} E+P M_{\chi_{\Omega \backslash \sigma}} E .
$$

Note that $P M_{\chi_{\sigma}}$ is a compact operator since $P$ is an integral operator with smooth kernel away from the boundary $\partial \Omega$. Choose $\sigma$ so that $v(\Omega \backslash \sigma)<\varepsilon$. Then, for $\|f\|_{\infty} \leq 1$, we have

$$
\left\|P M_{\chi_{\Omega \backslash \sigma}} E f\right\|_{2}=\left\|P M_{f} \chi_{\Omega \backslash \sigma}\right\|_{2} \leq\left\|\chi_{\Omega \backslash \sigma}\right\|_{2}<\sqrt{\varepsilon}
$$

so that $\left\|P M_{\chi_{\Omega \backslash \sigma}} E\right\|<\sqrt{\varepsilon}$. Hence, $P E$ is a norm limit of compact operators.

From the viewpoint of quantum mechanics, it may be of interest to extend our results to "infinitely many complex variables" (see $[\mathbf{2}, \mathbf{1 5}]$ ). This extension appears to work and the results remain approximately the same. We expect to treat this problem in a subsequent note.

\section{REFERENCES}

1. V. Bargmann, On a Hilbert space of analytic functions and an associated integral transform, Comm. Pure Appl. Math. 14 (1961), 187-214.

2. $\_$, Remarks on a Hilbert space of analytic functions, Proc. Nat. Acad. Sci. U.S.A. 48 (1962), 199-204. 
3. F. A. Berezin, Covariant and contravariant symbols of operators, Math. USSR Izv. 6 (1972), 1117-1151.

4. _ Q Quantization, Math. USSR Izv. 8 (1974), 1109-1163.

5. __ Quantization in complex symmetric spaces, Math. USSR Izv. 9 (1975), 341-379.

6. C. A. Berger and L. A. Coburn, Toeplitz openators and quantum mechanics, J. Funct. Anal. 68 (1986), 273-299.

7. O. Bratteli and D. Robinson, Operator algebras and quantum statistical mechanics I, II, Springer, $1979,1981$.

8. A. Friedman, Partial differential equations of parabolic type, Prentice-Hall, Englewood Cliffs, N. J., 1964.

9. A. Grossmann, G. Loupias, and E. M. Stein, An algebra of pseudo-differential operators and quantum mechanics in phase space, Ann. Inst. Fourier (Grenoble) 18 (1968), 343-368.

10. V. Guillemin, Toeplitz operators in n-dimensions, Integral Equations Operator Theory 7 (1984), 145-205.

11. R. Howe, Quantum mechanics and partial differential equations, J. Funct. Anal. 38 (1980), 188254.

12. S. C. Power, Commutator ideals and pseudo-differential $C^{*}$-algebras, Quart. J. Math. Oxford Ser. (2) 31 (1980), 467-489.

13. M. Rieffel, $C^{*}$-algebras associated with irrational rotations, Pacific J. Math. 93 (1981), 415-429.

14. I. E. Segal, Lectures at the Summer Seminar on Applied Math., Boulder, Col., 1960.

15. (1978), 321-343.

16. K. H. Zhu, VMO, ESV and Toeplitz operators on the Bergman space, Trans. Amer. Math. Soc. (to appear).

Department of Mathematics and Computer SCience, Herbert h. Lehman COllege, City UNiversity OF NEW YORK, BRONX, NEW YORK 10468

Department of Mathematics, State University of NeW York at Buffalo, BUfFALO, NEW YORK 14214 